\title{
Zonal flow dynamics and control of turbulent transport in stellarators
}

\author{
P. Xanthopoulos ${ }^{1}$, A. Mischchenko ${ }^{1}$, P. Helander ${ }^{1}$, H. Sugama ${ }^{2}$ and T.-H. Watanabe ${ }^{2}$ \\ ${ }^{1}$ Max-Planck-Institut für Plasmaphysik, Teilinstitut Greifswald, Wendelsteinstr. 1, D-17491 Greifswald, Germany \\ ${ }^{2}$ National Institute for Fusion Studies, Graduate University for Advanced Studies, Toki 509-5292, Japan
}

The relation between magnetic geometry and the level of ion-temperature-gradient (ITG) driven turbulence in stellarators is explored through gyrokinetic theory and direct linear and nonlinear simulations. It is found that the ITG radial heat flux is sensitive to details of the magnetic configuration that can be understood in terms of the linear behavior of zonal flows. The results throw light on the question of how the optimization of neoclassical confinement is related to the reduction of turbulence.

Understanding the turbulence present in tokamaks and stellarators is one of the most important challenges in plasma physics. A particularly interesting question in this context is how the magnetic geometry affects the nature and amplitude of the turbulence. In plasmas where the ion-temperature-gradient (ITG) instability is significant, so-called zonal flows (ZFs) have a favorable effect on the confinement [1]. However, the complexity introduced by nonaxisymmetry had rendered, only until very recently, the study of ZFs in stellarator configurations intractable. The dynamical character of the ZF response in a stellarator was first predicted analytically in Refs. $[2,3]$ and was later confirmed by linear gyrokinetic simulations $[4,5]$. In a tokamak, the linear response to an imposed ZF perturbation consists of geodesic acoustic mode (GAM) oscillations followed by a steady state so-called Rosenbluth-Hinton $(\mathrm{RH})$ residual level [6]. In stellarators, there is an intermediate stage of slow (compared with the GAM) damped ZF oscillations, and the $\mathrm{RH}$ residual level can be much lower than in tokamaks $[3,5]$. The details of this behavior depend on the magnetic configuration in question.

Recent work [7] sought to reduce turbulence in stellarators by directly targeting the ITG instability. Complementing that study, here we investigate the role of $\mathrm{ZF}$ dynamics in regulating turbulent transport. As will become apparent, the situation is different from that in tokamaks, since the residual level is not always reached before turbulence saturates (see Figs. 4,6), and in these cases, the RH level alone cannot account for differences in the heat flux (as sometimes claimed in the tokamak literature, see e.g., Ref. [8]). Furthermore, we identify the geodesic curvature as an important ingredient affecting the ZF oscillations, viz., minimizing the geodesic curvature proves to have a significant favorable effect on the confinement. Finally, we revisit the relationship between neoclassical (nc) optimization and turbulence reduction. Although these two concepts turn out to be correlated (see also Refs. [4,9]), here we show that turbulence must be treated in addition to nc optimization. It should be remembered that turbulent transport is important in optimized stellarators, especially at low temperatures [10].

As a preparatory step, to gain confidence in the numerical treatment, we present (the first published) linear and nonlinear benchmarks in stellarator geometry. Most of the presented simulations are performed with the GENE code (see, e.g., Refs. [11,12]), which solves the nonlinear, gyrokinetic system of equations in flux-tube geometry of an arbitrary toroidal configuration with closed flux surfaces. For the benchmarking, we compare GENE with the GKV code (see, e.g., Ref. [4]), which solves the same system of equations with independently developed numerical techniques. The instability considered is the electrostatic, collisionless ITG mode, assuming Boltzmann distributed (adiabatic) electrons.

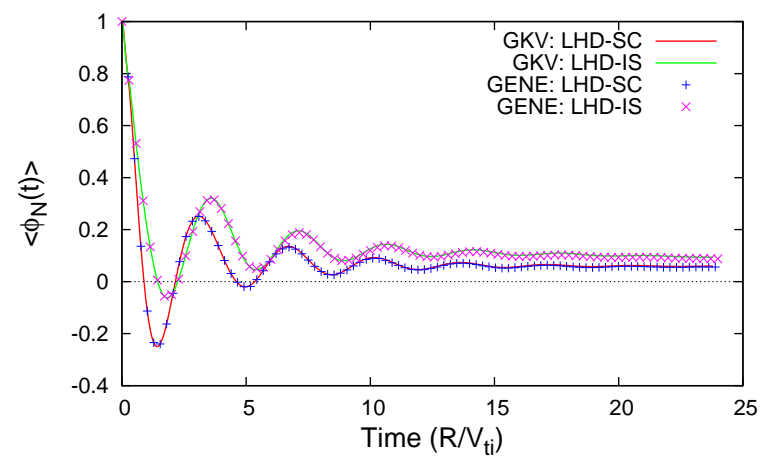

FIG. 1. Linear (normalized) ZF potential by GENE and GKV for two LHD configurations. Here, $V_{t i}=\sqrt{T_{i 0} / m_{i}}$ is the ion thermal velocity and $R$ is the major radius.

Starting with the linear case, we simulate two configurations of the Large Helical Device (LHD) [13], namely the standard (LHD-SC) and the inward-shifted (LHD-IS) configurations, to determine their linear ZF response. To this end, we have employed an analytical magnetic field representation in both codes (details of the implementation appear in Ref. [4]). The outcome of the comparison is shown in Fig. 1, where fast GAM oscillations are followed by a steady $\mathrm{RH}$ residual, resembling the response in a tokamak. For the nonlinear benchmarking, the computational effort is greatly increased. As inferred from Fig. 2, the two simulations for each LHD configuration are statistically almost equivalent at saturation. We note in passing that the discrepancy during the growing phase follows from the initial conditions of the codes (the lin- 
ear growth rates are in very good agreement). The differences between the two configurations seen in the two figures are believed to be related [4]: the fact that the LHD-IS configuration has a slower ZF decay and higher $\mathrm{RH}$ residual in the linear simulation leads to an increased averaged ZF amplitude in the nonlinear simulation and, in turn, to a reduced level of turbulence (even though the linear growth rates of the instability are a bit larger). Since this configuration also enjoys better nc confinement properties, it has been suggested that the suppression of turbulence is directly linked to the nc transport.

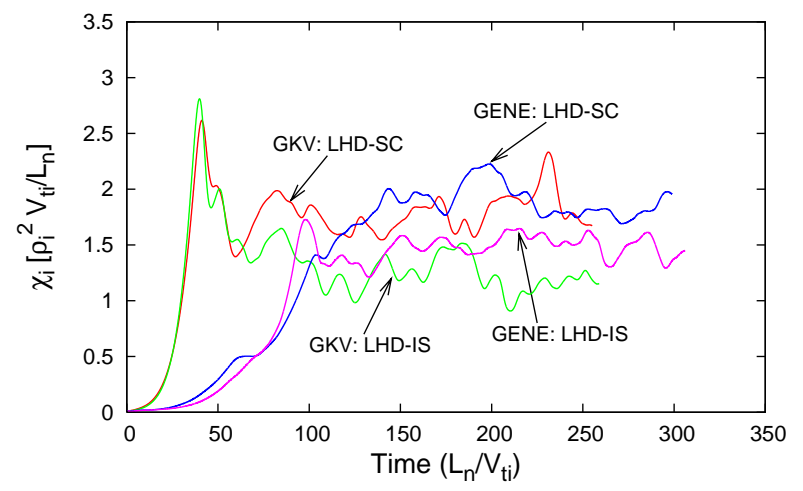

FIG. 2. Turbulent heat diffusivities from nonlinear ITG simulations produced by the GENE and GKV codes for two LHD configurations. $L_{n}$ denotes the density gradient scale length. The parameters of the simulations are described in Ref.[4].

As we shall see, the situation is more complicated. To understand why, we first provide a brief analytical treatment that explains the existence of $\mathrm{ZF}$ oscillations in a generic stellarator configuration [5]. The linear gyrokinetic equation for the ZF component of the electrostatic potential with perpendicular wavevector $\mathbf{k}_{\perp}=k_{r} \nabla r$ reads

$$
\frac{\partial f_{a 1}}{\partial t}+v_{\|} \nabla_{\|} f_{a 1}+i k_{r} v_{d} f_{a 1}=-i e_{a} k_{r} v_{d} \hat{\phi} J_{0}\left(k_{\perp} \rho\right) f_{0}
$$

where the zonal potential is normalized to the plasma temperature, i.e., $\hat{\phi}=e \phi / T, f_{0}$ is the Maxwellian equilibrium distribution function, $f_{1}$ is the perturbed gyrocenter distribution function, $J_{0}$ is the zeroth-order Bessel function, $\rho$ is the gyroradius, $v_{\|}$is the parallel velocity, and $v_{d}$ is the radial drift velocity. For simplicity, we assume $J_{0}\left(k_{\perp} \rho\right) \approx 1$ and equal ion and electron temperatures, although these restrictions can easily be relaxed.

Taking the first moment of Eq. (1) and employing the gyrokinetic quasineutrality condition, one obtains an equation for the evolution of the ZF potential

$$
n_{0}\left\langle k_{r}^{2} \rho_{i}^{2}\right\rangle \frac{\partial \hat{\phi}}{\partial t}+i k_{r} \sum_{a}\left\langle\int e_{a} v_{d} f_{a 1} \mathrm{~d}^{3} v\right\rangle=0
$$

Here, $e_{a}$ equals +1 for ions or -1 for electrons, and $n_{0}$ is the plasma density. The angular brackets denote fluxsurface averages.

Now, the initial-value problem is solved by applying the Laplace transform to Eq. (1) and assuming characteristic time scales much longer than the bounce time (taking $f_{i 1}(t=0)=\left\langle k_{r}^{2} \rho_{i}^{2}\right\rangle f_{0} \phi_{0}$ and $f_{e 1}(t=0)=0$ ). This allows us to cast Eq. (2) into the form of an ambipolarity condition $J_{r(p o l)}^{(c l)}+J_{r(p o l)}^{(n e o)}+J_{r(3 D)}=0$, with $J_{r(p o l)}^{(c l)}$ being the classical polarization current, $J_{r(\text { pol })}^{(\text {neo })}$ the nc polarization current and $J_{r(3 D)}=\sum_{a}\left\langle\int e_{a} \bar{v}_{d} f_{a 1} \mathrm{~d}^{3} v\right\rangle$ the radial current of locally trapped particles.

Solving this equation, we extract the ZF linear response on time scales longer than the bounce time (here $\left.\{\cdot\}:=\left\langle\int \cdot f_{0} \mathrm{~d}^{3} v\right\rangle\right)$

$$
\frac{\Phi(p)}{\phi_{0}}=\left\{\frac{1}{p+i \bar{\omega}_{d}}\right\} \frac{\left\langle k_{r}^{2} \rho_{i}^{2}\right\rangle}{\left\langle k_{r}^{2} \rho_{i}^{2}\right\rangle+\left\{k_{r}^{2} \delta_{r}^{2}\right\}+\left\{\frac{i \bar{\omega}_{d}}{p+i \bar{\omega}_{d}}\right\}}
$$

where $\Phi(p)$ is the Laplace transform of the potential, $\delta_{r}$ the radial orbit excursion, defined by $v_{d}=v_{\|} \nabla_{\|} \delta_{r}+\bar{v}_{d}$ and $\bar{\omega}_{d}=k_{r} \bar{v}_{d}$ is the bounce-averaged radial drift frequency. This equation is useful in two ways. First, the information about the existence of ZF oscillations follows from the dispersion relation

$$
\left\langle k_{r}^{2} \rho_{i}^{2}\right\rangle+\left\{k_{r}^{2} \delta_{r}^{2}\right\}+\left\{\frac{i \bar{\omega}_{d}}{p+i \bar{\omega}_{d}}\right\}=0
$$

and particularly the last term (in a tokamak $\bar{\omega}_{d}=0$ ).

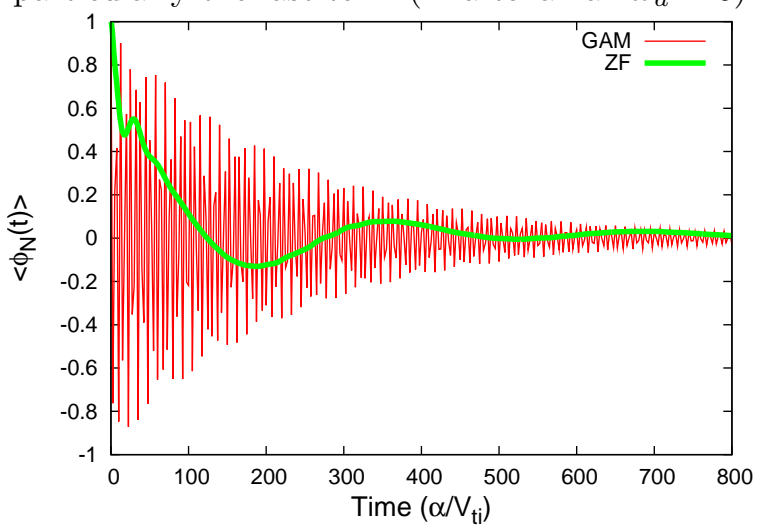

FIG. 3. Linear ZF potential for W7X for radial wavenumbers $k_{r} \rho_{i}=0.0005$, where GAM oscillations are pronounced, and $k_{r} \rho_{i}=0.06$, where ZF oscillations emerge. Here, $\rho_{i}=V_{t i} / \Omega_{i}$, where $\Omega_{i}$ is the ion gyrofrequency, and $\alpha$ denotes the (average) minor radius of the device.

The existence of these oscillations is also seen in Fig. 3, where a representative GENE result is shown for the Wendelstein 7-X (W7X) stellarator [14]. In fact, by selecting proper values for the radial wavenumber, it is formally possible to separate the fast GAM oscillations from the slow ones for the ZF response. Indeed, we observe strong GAMs but practically no ZF oscillations for small 
wavenumbers and vice versa for large ones (in the latter case, one can observe a small peak corresponding to the remnant GAM at the very beginning of the simulation). In addition, both the frequency and damping rate of the $\mathrm{ZF}$ oscillations are inherently related to the magnetic geometry (via $\left.\bar{\omega}_{d}\right)$.

Second, Eq. (4) can be used to calculate the RH residual level that is reached once the oscillations have died out, as

$$
\lim _{t \rightarrow \infty} \phi(t)=\lim _{p \rightarrow 0} p \Phi(p)=\frac{\left\langle k_{r}^{2} \rho_{i}^{2}\right\rangle\left(1-f_{h}\right) \phi(t=0)}{\left\langle k_{r}^{2} \rho_{i}^{2}\right\rangle+\left\{k_{r}^{2} \delta_{r}^{2}\right\}+f_{h}}
$$

where $f_{h}$ is the fraction of helically trapped particles, $f_{h}=\frac{1}{n_{0}}\left\langle\int_{k^{2}<1} \mathrm{~d}^{3} v f_{0}\right\rangle$ with $k^{2}$ the related trapping parameter. We remark that, in contrast to the tokamak case, the residual level is a function of the radial wavenumber (as was also reported in Ref. [2]), and is expected to vary among magnetic configurations. Specifically, when the impact of helically trapped particles is strong, i.e., $f_{h} \gg\left\{k_{r}^{2} \delta_{r}^{2}\right\}$, the residual level is very small, namely $\phi(t \rightarrow \infty) \approx\left\langle k_{r}^{2} \rho_{i}^{2}\right\rangle$, which is the situation we consider in the W7X case. In a less extreme case, where $f_{h} \sim\left\{k_{r}^{2} \delta_{r}^{2}\right\}$ holds, the resulting dependence of the residual level on $k_{r}$ is approximately linear (see Ref. [15] for an application in the LHD device).

How does the linear ZF dynamics thus considered affect the turbulent transport in terms of the radial heat flux levels? To address this issue, we carry out a numerical experiment, using the standard W7X configuration as a test bed and artificially reducing the geodesic curvature to a tenth of its nominal value throughout the simulated flux tube, without otherwise altering the fraction of helically trapped particles. Although the resulting magnetic configuration, termed here $\mathrm{W} 7 \mathrm{X} 10 \%$, is fictitious, this setup provides valuable insight, since the two configurations are characterized by exactly the same linear growth rates, something which is difficult to achieve through a self-consistent equilibrium calculation.

The GENE simulations reveal that the linear ZF responses are very different, as it is anticipated theoretically from the fact that the radial drift velocity, which is proportional to the geodesic curvature, enters in Eq. (3) for the linear ZF dynamics. This is illustrated by Fig. 4, where the configuration with small geodesic curvature is seen to exhibit a much slower decay of the initial perturbation. In a nonlinear simulation, this has the effect of boosting the average ZF amplitude, as shown in Fig. 5, and subsequently reducing the turbulent heat flux (see Fig. 6). It is important to note that this difference in the heat fluxes cannot be attributed to the residual level since, according to Eq. (5), its value for both configurations is extremely small, namely of the order $\left\langle k_{r}^{2} \rho_{i}^{2}\right\rangle$. Instead, in W7X, the ZF oscillations (similar to the ZF decay in LHD [4]), preceding the residual level, play a key role in the regulation of the turbulent transport.

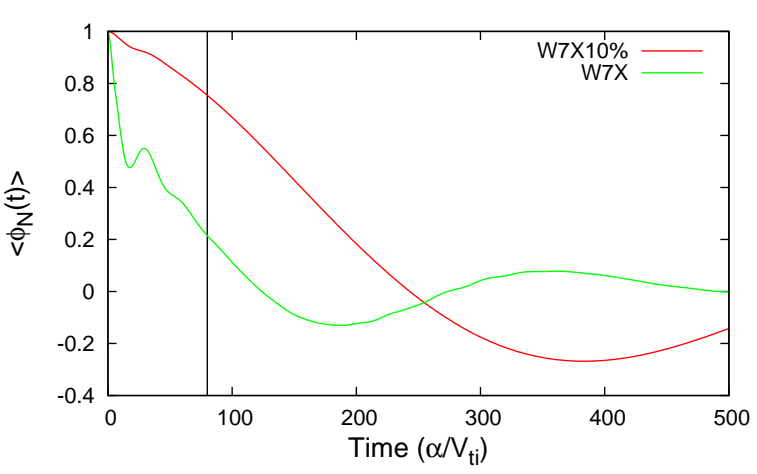

FIG. 4. Linear ZF responses for W7X and W7X10\% (see text). The vertical line denotes roughly the saturation time of turbulence, shown in Fig. 6.

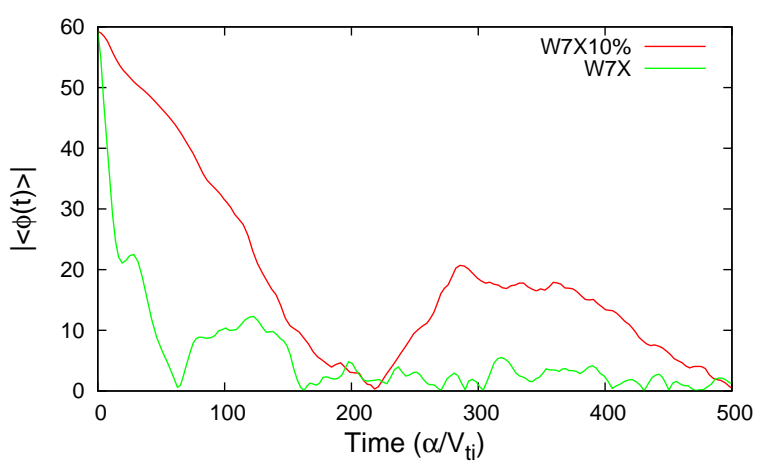

FIG. 5. ZF time traces from nonlinear ITG simulations for W7X and W7X10\% (see text).

On the other hand, reducing the geodesic curvature also has the known effect of shortening the radial step length in the nc transport. Therefore, the minimization of geodesic curvature reduces both the nc and turbulent transport channels, and therefore results in a correlation between nc optimization and turbulence reduction. This conclusion is a prominent candidate for the interpetation of the LHD cases studied in Ref. [4] (see also Figs. 1,2).

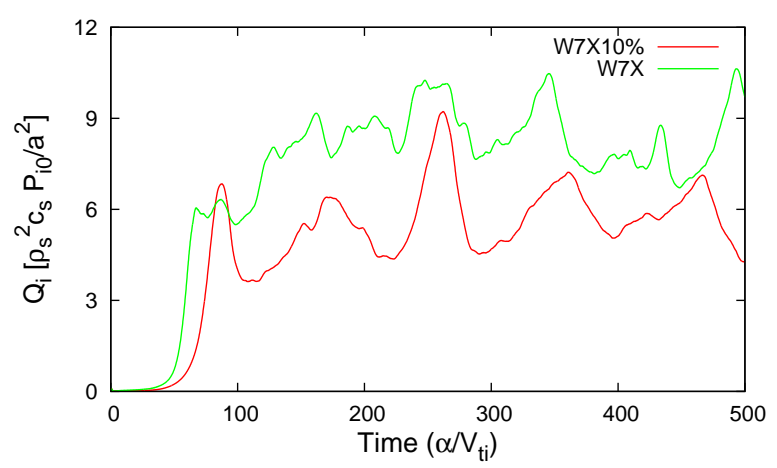

FIG. 6. Turbulent heat flux levels from nonlinear ITG simulations for W7X and W7X10\% (see text). 
Finally, we consider another member of the family of W7X configurations, the so-called Low Mirror Configuration (W7X-LM), which has larger helical ripple (about a factor of 3) than the standard configuration in the outer region of the plasma [16] but practically the same geodesic curvature. The linear ZF responses, in Fig. 7, are found to be very similar with respect to their decay (and the residual level is also very small). In addition, the linear ITG growth rates for the two simulations are essentially identical.

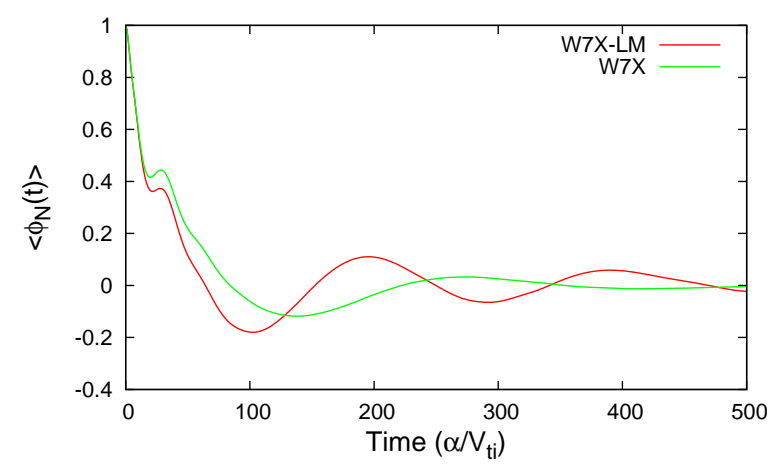

FIG. 7. Linear ZF responses for W7X and W7X-LM at radius $\rho=0.8 \alpha$.

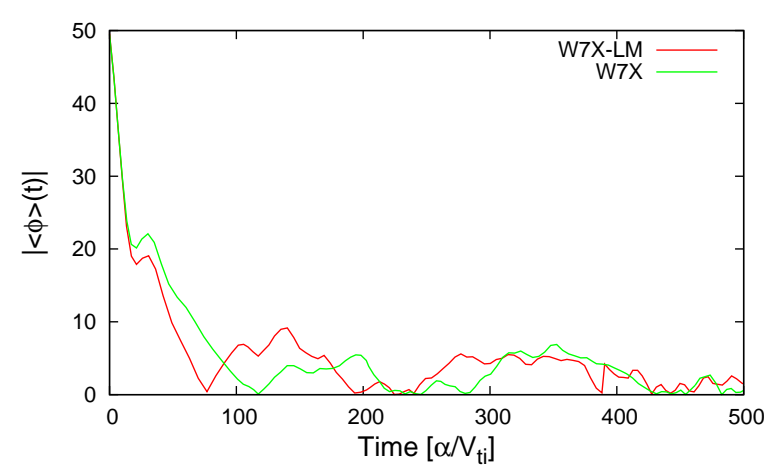

FIG. 8. ZF time traces from nonlinear ITG simulations for W7X-LM and W7X at $\rho=0.8 \alpha$.

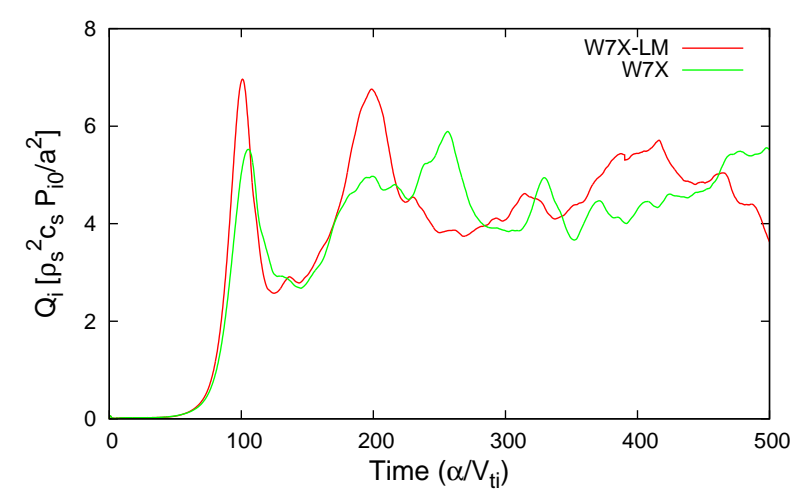

FIG. 9. Turbulent heat flux levels from nonlinear ITG simulations for W7X and W7X-LM at $\rho=0.8 \alpha$.
The nonlinear ZF amplitudes are consistent with the linear dynamics, as no significant difference is to be seen in Fig. 8 (compared to Fig. 5). Accordingly, the outcome of nonlinear ITG simulations for the two configurations, in Fig. 9, suggests that the levels are almost the same.

We conclude that the 3D magnetic field geometry affects the ITG heat transport via the linear ZF dynamics - oscillations in W7X or slow decay in LHD [4] prior to the $\mathrm{RH}$ residual level. An improved nc confinement is clearly correlated with reduced turbulent transport via the geodesic curvature. However, we demonstrated that configurations with very different effective ripple can have similar turbulent heat levels. Separate work [7], complementing this one, addressed the ITG instability, by minimizing regions of bad curvature. There, configurations with drastically reduced turbulent levels were generated, without affecting nc transport. Thus, the turbulent channel should be explicitly treated in future stellarator designs, in addition to nc optimization. We thank Prof. F. Jenko and Dr. C. Beidler for insightful comments and the Jülich Supercomputing Center for the GENE simulations.

[1] P. H. Diamond, S.-I. Itoh, K. Itoh and T. S. Hahm, Plasma Phys. Contr. Fusion 47, R35-R161 (2005)

[2] H. Sugama and T.-H. Watanabe, Phys. Plasmas 13, 012501 (2006)

[3] A. Mishchenko, P. Helander, and A. Koenies, Phys. Plasmas 15, 072309 (2008)

[4] T.-H. Watanabe, H. Sugama and S. Ferrando-Margalet, Phys. Rev. Lett. 100, 195002 (2008) ; Nucl. Fusion, 47, 1383 (2007)

[5] P. Helander, A. Mishchenko, R. Kleiber and P. Xanthopoulos, Plasma Phys. Contr. Fusion 53, 054006 (2011)

[6] M. Rosenbluth and F. Hinton, Phys. Rev. Lett. 80, 724 (1998)

[7] H. E. Mynick, N. Pomphrey and P. Xanthopoulos, Phys. Rev. Lett., 105, 095004 (2010)

[8] A. M. Dimits et al., Phys. Plasmas 7, 969 (2000)

[9] H. E. Mynick and A. Boozer, Phys. Plasmas 14, 072507 (2007)

[10] M. Hirsch et al., Plasma Phys. Contr. Fusion 50, 053001 (2008)

[11] F. Jenko, W. Dorland, M. Kotschenreuther, and B. N. Rogers, Phys. Plasmas 7, 1904 (2000)

[12] P. Xanthopoulos et al., Phys. Plasmas 16, 082303 (2009)

[13] O. Motojima, N. Ohyabu, A. Komori et al., Nucl. Fusion 43, 1674 (2003)

[14] J. Nührenberg and R. Zille, Phys. Lett. A 114, 129 (1986)

[15] H. Sugama and T.-H. Watanabe, Contrib. Plasma Phys., 50, $571(2010)$

[16] C. D. Beidler and H. Maassberg, Plasma Phys. Contr. Fusion 43, 1131-1148 (2001) 